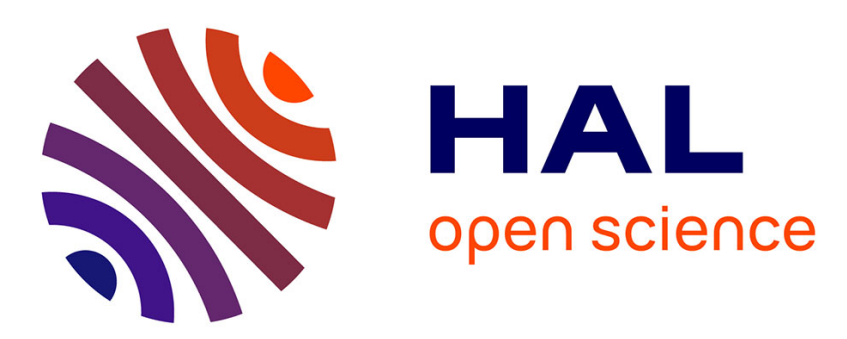

\title{
Partenariat, vassalité, marché et seigneurie: 4 configurations de contrôle client-fournisseur
}

Carole Donada, Gwenaëlle Nogatchewsky

\section{To cite this version:}

Carole Donada, Gwenaëlle Nogatchewsky. Partenariat, vassalité, marché et seigneurie : 4 configurations de contrôle client-fournisseur. Comptabilité - Contrôle - Audit, 2008, 14 (1), pp.145-168. halshs-00340597

\section{HAL Id: halshs-00340597 https://shs.hal.science/halshs-00340597}

Submitted on 8 Jul 2010

HAL is a multi-disciplinary open access archive for the deposit and dissemination of scientific research documents, whether they are published or not. The documents may come from teaching and research institutions in France or abroad, or from public or private research centers.
L'archive ouverte pluridisciplinaire HAL, est destinée au dépôt et à la diffusion de documents scientifiques de niveau recherche, publiés ou non, émanant des établissements d'enseignement et de recherche français ou étrangers, des laboratoires publics ou privés. 


\section{PARTENARIAT, VASSALITE, MARCHE ET SEIGNEURIE : 4 CONFIGURATIONS DE CONTROLE CLIENT-FOURNISSEUR}

Carole Donada, Professeur Associé, ESSEC, Av Bernard Hirsch, 95021 Cergy-Pontoise, 01.34.43.30.00, donada@essec.fr

Gwenaëlle Nogatchewsky, Maître de conférences, DRM-CREFIGE, CNRS UMR 7088, Université Paris Dauphine, Place du Maréchal de Lattre de Tassigny 75775 Paris cedex 16, 01 440547 43, nogatchewsky@voila.fr

\section{Résumé}

L'article présente les résultats d'une recherche sur le contrôle client-fournisseur en milieu industriel à partir de six études de cas. Quatre configurations de contrôle sont proposées en fonction de l'environnement achat des entreprises clientes. Pour chaque configuration, une organisation interne (structure achat et contrôle des acheteurs) et un contrôle sur les fournisseurs sont présentés. L'étude révèle également la coexistence de plusieurs modes de contrôle client-fournisseur pour une même configuration.

Mots clés : configurations de contrôle - relation client-fournisseur - dépendance
Abstract: Partnership, vassalage, market, and lordship: 4 buyer-supplier configurations

This article presents the results of a study on buyersupplier control in the manufacturing industry based on six case studies. Four control configurations are proposed according to the purchasing environment of buyers. For each configuration, an internal organisation (in terms of structure and internal control of purchasing agents) and a control of the buyer on its suppliers are presented. The study also reveals the coexistence of several modes of buyer-supplier control for a single configuration

Keywords: control configurations - buyer-supplier relationship - dependence 


\section{Introduction}

Les enjeux de l'externalisation pour les entreprises clientes sont considérables. D'une part, les achats représentent souvent près de $70 \%$ du coût de revient des produits dans l'industrie et, d'autre part, les entreprises sont souvent impliquées de manière quasi-irréversible avec des fournisseurs auxquels elles confient la conception et la réalisation de leurs composants stratégiques. Dès lors, on comprend la nécessité pour les entreprises clientes d'organiser le contrôle de leurs fournisseurs afin que ces derniers agissent conformément à leurs attentes.

Les travaux sur le contrôle client-fournisseur se sont multipliés ces dernières années (Dekker, 2004 ; Dumoulin, 1997 ; Hakansson et Lind, 2004 ; Langfield-Smith et Smith, 2003 ; van der Meer-Kooistra et Vosselman, 2000). Ils montrent l'influence des caractéristiques des transactions et des relations sur les modes de contrôle choisis par les entreprises clientes. Ces travaux sont riches d'enseignements pour le contrôle interorganisationnel, mais leurs auteurs appellent à de nouvelles recherches sur au moins deux aspects. D'une part, ils suggèrent d'élargir l'unité d'analyse de l'environnement au-delà de son caractère transactionnel et relationnel. Ils proposent de considérer l'influence de l'environnement institutionnel et des rapports de force sur le design du contrôle inter-organisationnel (Fabre, 2006 ; van der MeerKooistra et Vosselman, 2000). Il s'agit concrètement de distinguer les diverses situations de dépendance entre les clients et les fournisseurs pour comprendre les rapports de pouvoir et leurs effets sur les organisations en place et les stratégies poursuivies. D'autre part, certains chercheurs regrettent que les entreprises étudiées soient considérées comme des «boîtes noires » (Hakansson et Lind, 2004). Ils reconnaissent l'implication d'un grand nombre d'acteurs au sein de chaque organisation dans la conduite des relations interorganisationnelles, acteurs dont les motivations peuvent être diverses voire contradictoires. Dès lors, ils invitent les chercheurs à examiner les caractéristiques internes des organisations en relation, ces caractéristiques pouvant influencer les modalités du contrôle interorganisationnel.

L'objet de l'article est d'intégrer ces préoccupations afin de mieux comprendre comment les entreprises s'organisent et modulent leur organisation pour contrôler leurs fournisseurs, 
compte tenu de leur situation de dépendance client-fournisseur. Dans la lignée des travaux sur le contrôle organisationnel de Flamholtz (1996), le contrôle du client sur son fournisseur est défini comme étant le processus par lequel le client influence les actions du fournisseur dans le sens de ses attentes. Ce contrôle s'exerce dans un environnement achat dont la structure peut être appréhendée par le niveau de dépendance entre les entreprises clientes et leurs fournisseurs. Et, en portant notre attention sur l'organisation interne de l'entreprise cliente, nous examinons la structure de l'organisation achat sur deux aspects : la structure formelle (l'organigramme) et les mécanismes de contrôle interne des responsables en titre de la relation avec les fournisseurs (les acheteurs).

Cette recherche s'appuie sur six études de cas d'entreprises clientes issues de divers secteurs industriels : automobile, aéronautique, pharmaceutique, agro-alimentaire et télécommunications. Chaque cas retenu s'inscrit dans un environnement achat particulier en termes de dépendance réciproque avec ses fournisseurs. L'analyse des cas nous permet d'identifier quatre configurations de contrôle client-fournisseur : le partenariat, la vassalité, le marché et la seigneurie. Pour chaque configuration, nous observons une cohérence entre l'environnement achat, l'organisation de l'entreprise cliente et le contrôle que celle-ci exerce sur ses fournisseurs. L'étude révèle également la coexistence de plusieurs modes de contrôle pour une même configuration client-fournisseur.

L'article est structuré en deux parties. La première partie dresse le cadre théorique et empirique de la recherche. La seconde partie présente quatre configurations du contrôle client-fournisseur.

\section{Cadre de la recherche}

La présentation du cadre de cette recherche commence par une revue de la littérature sur le contrôle interorganisationnel et les approches théoriques permettant de le comprendre. La méthodologie développée pour mener à bien l'étude empirique présentée ici est exposée dans une seconde section. 


\subsection{Revue de la littérature}

L'objectif de cette revue est d'examiner comment le contrôle interorganisationnel est traité dans la littérature, de noter les manques et de proposer un nouveau cadre d'analyse pour étudier l'organisation du contrôle client-fournisseur. Cette revue est structurée en deux parties. La première rappelle les deux principales approches théoriques du contrôle interorganisationnel et leur intégration nécessaire. La seconde partie traite de la pertinence à considérer aussi la dépendance entre les entreprises clientes et fournisseurs ainsi que les modalités de leur organisation interne.

\subsubsection{Le contrôle inter-organisationnel dans la littérature}

Deux approches sont principalement mobilisées pour traiter du contrôle interorganisationnel : l'approche transactionnelle et l'approche relationnelle. Ces approches sont souvent intégrées dans des typologies proposant trois modes de contrôle (Donada et Nogatchewsky, 2006 ; Petitjean, 2001).

\section{Modalités du contrôle dans l'approche transactionnelle}

Au sein de l'approche transactionnelle, la théorie des coûts de transaction (TCT) rassemble les auteurs qui analysent les échanges comme étant des transactions économiques (Williamson, 1985). Toute transaction génère un coût -issu de la confrontation des agents- qui vient s'ajouter au seul coût du produit échangé. Ce coût dépend des caractéristiques des transactions (incertitude, fréquence, actifs spécifiques). Il est également déterminé par des facteurs humains (rationalité limitée et opportunisme) et environnementaux (nombre d'acteurs sur le marché, incertitude). Dans ce cadre, il convient de choisir la structure de gouvernance qui permet la gestion la plus efficiente des transactions. Trois formes de gouvernance auxquelles sont associées différents types de contrat sont proposées :

- le marché qui s'appuie sur le contrat classique (contrat détaillé et complet).

- la forme hybride qui s'appuie sur le contrat néo-classique dans lequel chaque partie accepte l'assistance d'une tierce partie (un arbitre) dans la résolution des conflits et l'évaluation de l'exécution ;

- la hiérarchie qui requiert un contrat personnalisé et des mécanismes hiérarchiques tels que l'autorité (le fiat). 
Les modes de gouvernance proposés -à travers les différents contrats- visent à réduire l'incertitude de chaque partie et à se prémunir contre l'opportunisme éventuel de l'autre.

$\mathrm{Au}$ sein de l'approche transactionnelle, la théorie de l'agence rassemble les auteurs qui analysent les mécanismes d'incitation et de protection des parties dans un contexte d'agence (Jensen et Meckling, 1976). Deux types de contrat entre un principal et un agent peuvent être proposés :

- un contrat sur les comportements (procédures à suivre) ;

- un contrat sur les résultats (objectifs à atteindre).

Dans le cadre de relations interentreprises, ces contrats visent à gérer l'opportunisme éventuel de l'agent dans des situations d'incertitude et de divergence d'intérêts (Celly et Frazier, 1996). Plus le produit est complexe, l'environnement incertain et le fournisseur difficile à remplacer, plus le client doit privilégier un contrôle des comportements plutôt qu'un contrôle des résultats.

Cette approche transactionnelle se traduit par deux modes de contrôle dans les relations interentreprises : un contrôle par le marché qui porte sur les résultats à court terme et un contrôle plus hiérarchique ou bureaucratique qui met aussi l'accent sur les comportements.

\section{Modalités du contrôle dans l'approche relationnelle}

A la logique transactionnelle des échanges, l'approche de l'échange relationnel oppose la prise en compte du contexte historique et social dans lequel s'inscrivent les épisodes de la relation. S'appuyant sur la théorie de l'échange social (Blau, 1964, Thibaut et Kelley, 1959), sur les travaux de Macaulay (1963) relatifs aux relations non contractuelles et sur ceux de Macneil (1980) concernant la distinction entre l'échange discret et l'échange relationnel, les chercheurs étudient les mécanismes de contrôle, plus sociaux qu'économiques, qui gouvernent les échanges et améliorent l'efficacité de la coopération (Larson, 1992). Ces mécanismes évoluent conjointement au développement de la coopération selon des phases successives (Dwyer et al., 1987 ; Jap et Ganesan, 2000 ; Larson, 1992 ; Ring et van de Ven, 1992, 1994). Au début, la coopération porte sur des opérations peu risquées et s'appuie sur des contrats classiques. Puis, le succès des premiers échanges permet aux partenaires de mieux se connaître, les incite à s'engager dans des opérations plus risquées et à approfondir la coopération. Dans cette phase de construction et de maturité de la relation, le contrôle du 
partenaire ne passe plus nécessairement par des contrats formels. D’autres mécanismes prennent le relais. Les interactions répétées entre les individus font émerger des règles explicites (procédures de communication, règles de partage de l'information) et implicites (honnêteté, transparence, équité, réciprocité) qui consolident la coopération (Larson, 1992). Ces règles sont en fait des normes relationnelles qui, dans un climat de confiance, structurent les échanges et procurent la coordination nécessaire à leur bon déroulement. Ainsi, le contrôle informel remplace progressivement les dispositifs formels (Ring et van de Ven, 1994). Ce mode de contrôle, appelé contrôle social ${ }^{1}$, est avant tout un moyen de se coordonner et d'atteindre des buts communs à long terme dans une perspective où ce qui unit les parties (les projets communs) est plus fort que ce qui peut les diviser.

Intégration des approches transactionnelle et relationnelle dans trois modes de contrôle: prolongements

La plupart des recherches empiriques sur le contrôle inter-organisationnel s'appuient sur ces deux approches (transactionnelle et relationnelle) pour établir une typologie en trois modes de contrôle (Dekker, 2004 ; Donada et Nogatchewsky, 2006 ; Dumoulin, 1997 ; Dumoulin et Gbaka, 1997 ; Hakansson et Lind, 2004 ; Langfield-Smith et Smith, 2003 ; van der MeerKooistra et Vosselman, 2000) : le marché, la bureaucratie (ou hiérarchie) et un troisième mode appelé contrôle social, par la confiance ou $\operatorname{clan}^{2}$. Ces modes de contrôle peuvent être analysés selon les phases de la relation. Van der Meer-Kooistra et Vosselman (2000) en distinguent trois : le contact, la contractualisation et l'exécution. Les deux premières ont en fait trait aux modalités de sélection des fournisseurs (recherche de partenaires et formalisation de la coopération).

Les contributions de ces recherches sont riches d'enseignements. Elles appellent néanmoins des prolongements sur deux aspects (van der Meer-Kooistra et Vosselman, 2000) : la prise en compte de la dépendance entre les parties et les modalités de leur organisation interne.

- Dans les approches relationnelles et transactionnelles, les problématiques de pouvoir et de dépendance sont peu considérées. Ces questions étaient très présentes dans les travaux des années 70-80 (Pfeffer et Salancik, 1978 ; Pfeffer, 1981). Puis, dans les années 80-90, les préoccupations des chercheurs se sont déplacées sur les modalités de coordination inter-organisationnelles dans des contextes où les entreprises, fortement interdépendantes dans le temps, devaient coopérer pour se développer ensemble et non 
les unes contre les autres (Aoki, 1991). Aujourd'hui, un certain nombre de chercheurs suggèrent de réintégrer les notions de dépendance et de rapports de force qui, même dans les coopérations de long terme, influencent fortement les risques d'opportunisme des partenaires aux différents stades de leur relation (Jap et Ganesan, 2000).

- Les entreprises en relation sont souvent considérées comme des «boîtes noires » dont les modalités d'organisation interne sont négligées. Or, la compréhension des relations client-fournisseur ne peut s'exonérer d'une compréhension des mécanismes de coordination interne (Anderson et al., 1994). Les travaux de van der Meer-Kooistra (2000) et Hakansson et Lind (2004) mentionnent l'influence des structures internes sur le contrôle interentreprises et invitent les chercheurs à approfondir leurs recherches dans ce sens.

Dans cette perspective, nous proposons d'intégrer les problématiques de dépendance et d'explorer les modalités d'organisation interne des entreprises (en termes de structure formelle et de modalités de contrôle interne) dans cette étude sur le contrôle clientfournisseur. L'approche par le pouvoir et la dépendance, largement popularisée par Pfeffer et Salancik (1978) offre un cadre tout à fait pertinent pour l'analyse des relations interorganisationnelles (Donada et Nogatchewsky, 2006).

\subsubsection{La prise en compte de la dépendance et de l'organisation interne sur le contrôle client-fournisseur}

\section{L'importance de la dépendance client-fournisseur}

Selon Emerson (1962, p. 32), «la dépendance d'un acteur $A$ sur un acteur B est (1) directement proportionnelle à la motivation de A pour les enjeux contrôlés par B et (2) inversement proportionnelle à la disponibilité de ces enjeux en dehors de la relation $A-B »$.

Considérant que les organisations sont dépendantes de leur environnement puisqu'elles y puisent les ressources dont elles ont besoin, Pfeffer et Salancik (1978) ont développé une théorie de la dépendance des ressources. Ils examinent le niveau de dépendance d'une organisation sur une autre à travers l'importance de la ressource échangée, le nombre d'acteurs qui la contrôlent et l'étendue du pouvoir de celui qui la détient. Pfeffer et Salancik (1978) montrent comment les organisations qui cherchent à réduire leur incertitude et leur 
dépendance sur les ressources transforment et négocient leur environnement par des relations avec leurs partenaires d'échanges. Considérant également l'importance de la dépendance dans les relations inter-entreprises, Ulrich et Barney (1984) insistent sur la nécessité de coordonner les échanges par la mise en place de liens formels. D'autres chercheurs mettent en avant les stratégies d'influence coercitives (menaces, arguments juridiques ou promesses) et non coercitives (requêtes, recommandations, demandes d'information) pour orienter les décisions des partenaires (Frazier et Rody, 1991 ; Gundlach et Cadotte, 1994 ; Kim, 2000). Ces études examinent aussi l'importance de l'amplitude et de l'asymétrie de dépendance dans les relations client-fournisseur. Toutefois, leurs auteurs limitent encore le contrôle interorganisationnel à la mise en place de structures de gestion reposant sur des liens formels (en particulier juridiques) et à l'expression d'une coercition vis-à-vis d'un partenaire. Or, comme Bouquin (2006) le suggère, le contrôle interorganisationnel est un processus beaucoup plus complexe qui, à l'instar du contrôle organisationnel, vise à assurer une cohérence entre l'organisation et son environnement.

\section{Le rôle de l'organisation interne dans le contrôle client-fournisseur}

Dans la littérature en management, l'organisation interne est souvent appréhendée par sa structure. Selon Desreumaux (1992, p. 50), la structure est définie comme « une allocation relativement stable de tâches et de rôles créant un pattern d'activités interreliées et permettant à l'entreprise de conduire et de coordonner ses activités ». La structure d'une organisation conditionne les choix et les comportements des individus qui la composent. Dès lors, la structure achat d'une entreprise cliente devrait conditionner les choix et les comportements des acheteurs vis-à-vis de leurs fournisseurs. Suivant Mintzberg (1982), les auteurs appréhendent généralement la structure d'une organisation par sa forme et ses mécanismes de coordination ou de contrôle. Dans ce cadre, la structure achat d'une entreprise cliente est étudiée à travers sa forme (organigramme) et les mécanismes de contrôle interne des acheteurs qui sont en charge de la relation avec les fournisseurs.

La forme d'une organisation achat peut être appréhendée par son degré de centralisation et par l'existence ou non de fonctions support. Nous suggérons alors que le positionnement des équipes achat, leurs processus de décision et leur influence dans ces processus peuvent déterminer le contrôle que l'organisation cliente (par ses différents acteurs) met en œuvre auprès de ses fournisseurs. De même, la localisation du (ou des) département(s) achat au sein 
de l'entreprise, l'existence ou non d'une fonction qualité dédiée aux achats, ainsi que les liens que le département achat entretient avec les autres fonctions de l'entreprise (qualité, ingénierie, production) peuvent influencer ce contrôle.

Les mécanismes de contrôle interne des acheteurs sont l'ensemble des dispositifs qui orientent les comportements des acheteurs, notamment vis-à-vis de leurs fournisseurs. Définis par la direction des achats, ces mécanismes reflètent les attentes de l'entreprise cliente à l'égard des acheteurs. Ces attentes s'expriment sur des résultats et / ou sur des comportements. Elles peuvent se traduire par des définitions d'objectifs et des modalités d'évaluation des performances des acheteurs.

En définitive, l'analyse de la littérature sur le contrôle interorganisationnel met en évidence trois modes de contrôle (marché, bureaucratie, social) qui s'appuient sur les approches transactionnelle et relationnelle. Dans l'étude du contrôle client-fournisseur, ces modes peuvent être appréhendés selon deux phases (la sélection et l'exécution). Ils doivent prendre en compte les effets de la dépendance entre les entreprises clientes et leurs fournisseurs. Ils doivent également intégrer la structure des entreprises en relation et, en particulier, celle de l'entreprise cliente.

\subsection{Etude empirique}

L'objectif de cette section est de présenter la méthodologie par étude de cas et de représenter l'environnement achat des entreprises clientes.

\subsubsection{Méthodologie}

Cette recherche s'appuie sur six études de cas réalisées auprès d'entreprises clientes de différents secteurs industriels: automobile (Autoplus et Consauto), aéronautique (Airindustrie), pharmaceutique (Pharmacop), agro-alimentaire (Agro) et télécommunications (Telecom). Le contrôle client-fournisseur qui intègre l'organisation de l'entreprise cliente étant un objet de recherche complexe et peu étudié, il était préférable d'explorer ce champ en procédant à une analyse empirique qualitative (Yin, 1989).

Chaque cas retenu pour cette recherche s'inscrit dans un environnement achat particulier en termes de dépendance réciproque entre le client et ses fournisseurs. Sur treize entreprises industrielles contactées, dix évoluaient dans un environnement achat caractéristique de 
diverses situations d'amplitude et d'asymétrie de dépendance client-fournisseur. Parmi ces dix cas potentiels, trois entreprises n'ont pas souhaité participer à une étude approfondie compte tenu du temps requis et une autre a connu de graves difficultés financières (mise en liquidation), ce qui a interrompu l'étude. Nous avons donc retenu six cas. Leur étude a duré dix-huit mois au cours desquels nous avons interrogé quarante acheteurs au sein de ces entreprises clientes et quatorze de leurs fournisseurs. Les entretiens avec les acheteurs ont porté sur trois thèmes :

- l'environnement achat de l'entreprise cliente ;

- l'organisation achat de l'entreprise cliente : structure achat et modalités de contrôle des personnes en charge de la relation client-fournisseur (attentes à l'égard des acheteurs, objectifs, pilotage, évaluation, sanctions-récompenses) ;

- le contrôle des fournisseurs : modalités de sélection des fournisseurs puis choix du fournisseur le plus représentatif du portefeuille; évaluation de la dépendance réciproque avec ce fournisseur; modalités de contrôle de ce fournisseur (attentes, objectifs, pilotage, évaluation, sanctions-récompenses, modalités relationnelles, réactions des fournisseurs).

Parallèlement, nous avons interrogé des fournisseurs de chaque entreprise sur les dispositifs de contrôle du client, les modalités relationnelles entre les équipes et les réactions des fournisseurs à l'égard de leur client. Enfin, nous avons consulté et étudié des documents internes aux entreprises (e.g. tableaux de bord de suivi des fournisseurs, entretiens d'évaluation d'acheteurs, grilles d'évaluation des fournisseurs, processus achat en projet....) et externes (revue de presse, états financiers...). Toutes les données ont été analysées selon la méthode préconisée par Miles et Huberman (1991). Nous avons construit des catégories pertinentes a priori à partir de notre analyse de la littérature, catégories que nous avons complétées grâce aux données du terrain. Les données ont été codées grâce au logiciel NVivo, ce qui nous a permis d'élaborer des matrices intra-site puis inter-sites afin de faire émerger des schémas d'analyse.

\subsubsection{Matrice de l'environnement achat}

Interrogés sur leur environnement achat, les acheteurs insistent sur deux points : 1) l'importance des effets de la concentration des entreprises et des échanges et 2) le rôle déterminant des contraintes technologiques, industrielles, réglementaires. Retenant le fournisseur le plus représentatif de leur portefeuille, les acheteurs évaluent la dépendance 
réciproque de leur entreprise cliente face à celle du fournisseur à partir de trois items généralement utilisés par les chercheurs mesurant la dépendance interentreprises. Concrètement, nous avons repris les échelles validées en marketing Business to Business (Frazier et al., 1989 ; Heide, 1994 ; Lusch et Brown, 1996) pour évaluer la dépendance du client et la dépendance du fournisseur. Les acheteurs interrogés ont évalué trois items de 1 à 5 (de «pas du tout d'accord » à «tout à fait d'accord »).

Toutes les réponses ont permis de positionner les cas sur une matrice de dépendance présentée dans la figure 1. Les deux axes constitutifs de la matrice sont : le niveau de dépendance du client (forte/faible) et le niveau de dépendance des fournisseurs (forte/faible). Ce format de matrice rappelle celui adopté par les chercheurs en marketing industriel ou les consultants en management des achats (Cox, 2004).

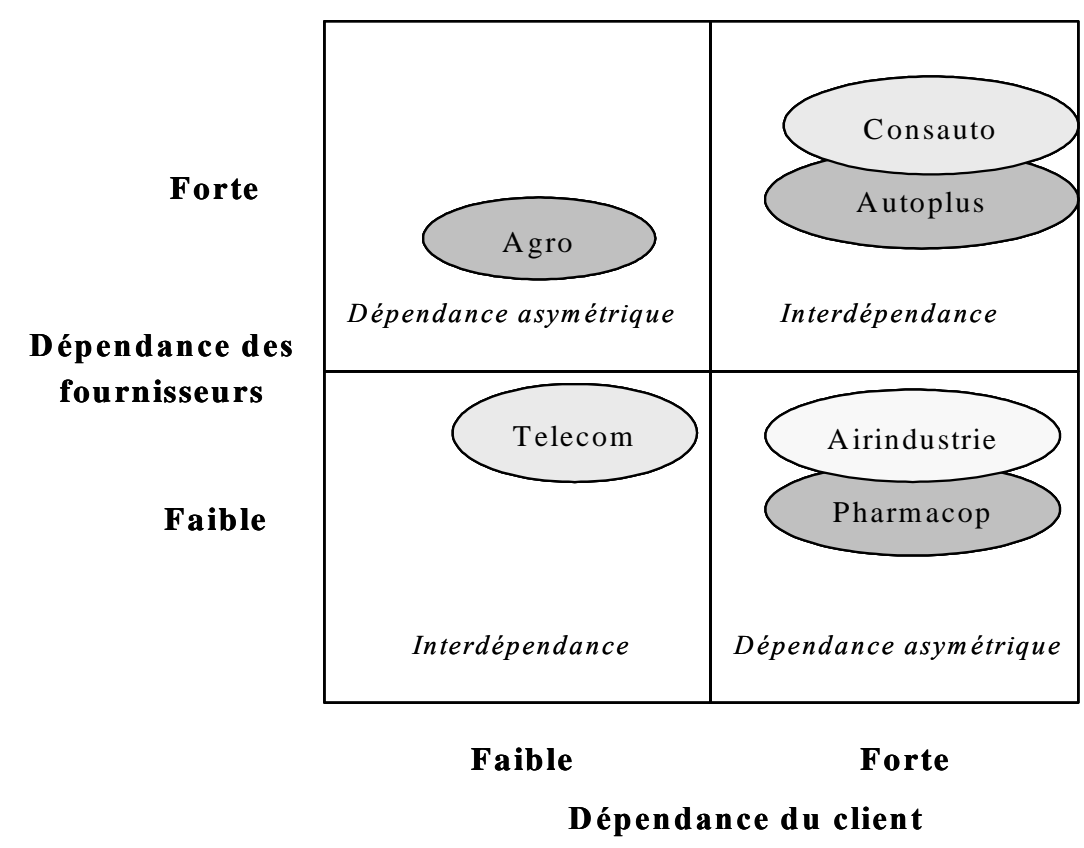

Figure 1 : Positionnement des cas dans leur environnement achat

\section{Quatre configurations de contrôle client-fournisseur}

L'analyse des cas présentés dans la matrice met en évidence quatre configurations de contrôle client-fournisseur : le partenariat, la vassalité, le marché et la seigneurie.

Les configurations du partenariat et du marché apparaissent lorsque les parties sont interdépendantes. Leur interdépendance est forte dans le cas du partenariat (cas Consauto et 
Autoplus); elle est faible dans celui d'une configuration par le marché (cas Telecom). En revanche, les configurations de la vassalité et de la seigneurie apparaissent en situation de dépendance asymétrique entre les parties. L'asymétrie est défavorable au client dans celle de la vassalité (cas Airindustrie et Pharmacop) ; elle est favorable à l'entreprise cliente dans la configuration de la seigneurie (cas Agro) $)^{3}$.

La présentation et l'analyse de chaque configuration suivent une même structure en trois points. Nous présentons pour chaque entreprise cliente : (1) les principales caractéristiques de son environnement achat, (2) son organisation achat (en termes de structure et de contrôle interne des acheteurs) et (3) le contrôle qu'elle opère sur ses fournisseurs aux deux moments clés d'une relation client-fournisseur : la sélection des fournisseurs et l'exécution du contrôle. La phase d'exécution est analysée sur tout son cycle, y compris ses suites. En analysant l'ensemble du processus de contrôle, nous suivons les recommandations des chercheurs qui suggèrent une analyse des différentes étapes d'une relation (Dwyer et al., 1987 ; Jap et Ganesan, 2000 ; Van der Meer-Kooistra et Vosselman, 2000).

\subsection{Les cas Consauto et Autoplus : une configuration de partenariat}

\subsubsection{Une forte interdépendance entre le client et le fournisseur}

Consauto et Autoplus sont deux constructeurs automobiles dont les achats représentent environ $70 \%$ du coût de revient de leurs véhicules. Les deux entreprises sont fortement liées à leurs fournisseurs équipementiers qui sont présents sur les plateaux-projets et implantés près des sites d'assemblage des véhicules. Le petit nombre de constructeurs et de fournisseurs de premier rang ainsi que la nécessaire collaboration entre tous les acteurs d'un projet de véhicule dès ses premières phases de conception créent une très forte interdépendance clientfournisseur.

\subsubsection{Une structure achat matricielle étoffée ; un contrôle des acheteurs formel et structuré} portant sur la stratégie achat, les résultats, les procédés de travail et les comportements à l'égard des fournisseurs

La structure achat des deux entreprises clientes Consauto et Autoplus est matricielle (projet/équipement) et très étoffée. Pour chaque projet de véhicule, les achats sont 
décentralisés et confiés à des acheteurs spécialisés dans chaque équipement (siège, tableau de bord, etc.). Les acheteurs projet interviennent dès les phases amont de la conception. Parallèlement, pour chaque type d'équipements, les achats sont centralisés sous l'autorité d'un responsable achat pour cette famille d'équipements. Ce dernier coordonne tous les acheteurs projet spécialisés dans son type d'équipements. En support, des experts en logistique, qualité, finance ou présence internationale évaluent les fournisseurs dans leur domaine respectif. Cette structure achat matricielle étoffée place les équipes achat au centre du processus de décision concernant les fournisseurs. Elle est cohérente avec l'environnement achat de Consauto et Autoplus (petit nombre de fournisseurs spécialisés sur les équipements demandés et obligation de maintenir des collaborations avec les fournisseurs sur tout le cycle de vie du projet): la structure décentralisée par projet facilite le partenariat interne entre toutes les parties prenantes d'un projet (acheteurs, bureaux d'études etc.). La structure centralisée par équipement permet d'exploiter les similitudes entre les projets et de profiter des effets volume.

Le contrôle des acheteurs de Consauto et d'Autoplus est formel et structuré. Il est spécifique à chaque type d'acheteurs.

Les acheteurs équipement sont évalués sur la formulation de leur stratégie achat concernant leur famille d'équipements, sur leurs résultats principalement en termes de gains sur achat et niveau de qualité des produits fournis (mesuré en $\mathrm{PPM}^{4}$ ). En charge du choix des fournisseurs sur les projets, ils doivent se coordonner avec les équipes projet et les experts. Le détail de leurs activités et les procédures à suivre vis-à-vis des fournisseurs sont inscrits dans un manuel.

Parallèlement, les acheteurs projet sont évalués sur leur capacité à préparer les fournisseurs dans les délais en respectant un prix et une qualité cibles. Ils sont en charge du suivi des quatre points QCDP (qualité, coût, délais, poids), de la mise en place du capacitaire et du suivi de projet (tableau de bord des risques, planning, investissements, points bloquants). Leur horizon temporel est uniquement celui du projet. Ils doivent en suivre les jalons selon des procédures strictes.

Tous les acheteurs (équipement et projet) étant in fine notés sur leurs résultats, ils pourraient être tentés d'obtenir le maximum d'économies à court terme en privilégiant des stratégies d'influence coercitives auprès de leurs fournisseurs sans tenir compte de l'interdépendance constructeur-équipementiers sur le très long terme. Pour prévenir ce risque, les directions des achats de Consauto et Autoplus demandent explicitement aux acheteurs de développer des 
comportements positifs à l'égard des fournisseurs (honnêteté, comportement éthique, respect des engagements).

Enfin, les experts dédiés à la direction achat doivent s'assurer du respect des critères dans leur domaine au moment de la formulation de la stratégie achat (et donc de la pré-sélection des fournisseurs) et au cours des projets.

\subsubsection{Un contrôle des fournisseurs très organisé associant dispositifs bureaucratiques et relationnels selon les acteurs de la relation}

La phase de sélection des fournisseurs est préparée lors de la stratégie achat qui définit les parts de marché globales accordées à chacun. Cette phase débute par une évaluation formelle des fournisseurs sur des critères prédéfinis ${ }^{5}$ : résultats (compétitivité prix, qualité, logistique), compétences ( $R \& D$, industrialisation), fit organisationnel et stratégique (stratégie marketing, développement international, pérennité et gouvernance), comportements (qualité du relationnel avec les équipes du client). La sélection repose donc sur un processus économicorationnel et un dispositif très structuré plutôt de facture bureaucratique, ce qui, d'après Dekker (2004), permet d'atténuer les problèmes de contrôle, de susciter la confiance au sens large ${ }^{6}$ et de favoriser ex ante l'encastrement entre les parties prenantes d'un projet. Toutefois, à l'instar de ce que décrivent Pezet (2001) pour les décisions d'investissement et Fabre (2006) pour les attributions de moyens, les choix des fournisseurs sont aussi enchevêtrés dans un jeu organisationnel politique entre des acteurs (e.g. entre les acheteurs, les ingénieurs des projets). Si l'évaluation formelle des ressources et capacités reste le pilier du contrôle chez Consauto et Autoplus, l'importance de chaque critère est parfois laissée à la discrétion des parties prenantes qui influencent les attributions de fournisseurs par projet (antériorité des relations entre les équipes client-fournisseur, capital social et relationnel entre les partenaires etc.). Les contrats qui appuient la sélection des fournisseurs sont certes détaillés mais incomplets ; ils prévoient des sanctions bilatérales en cas de défaillance.

Si la sélection correspond à un niveau stratégique de contrôle, le contrôle de la phase d'exécution s'opère à la fois à un niveau de gestion et à un niveau opérationnel. Cette situation fait écho aux trois niveaux de contrôle présentés par Anthony (1993).

Le contrôle de gestion est orchestré par les acheteurs équipement. Un peu mieux structuré chez Consauto que chez Autoplus, ce contrôle est, dans les deux cas, de facture bureaucratique. Les dispositifs mis en place sont complexes et nombreux. Ils portent sur les 
résultats (coûts, qualité, délais), les procédés de travail (qualité du process, méthodes de production) et sur les comportements (implication des fournisseurs). Ils s'appuient sur des systèmes formels d'information (tableaux de bord, reporting, audits, chantiers de productivité) et s'organisent autour des revues trimestrielles de performance. Ils s'appuient également sur des sources plus informelles (indiscrétions, confidences, visites des sites) qui complètent les informations sur les capacités réelles des fournisseurs, leur stratégie et leur rapport de force avec les constructeurs. L'évaluation de ce rapport de force, qui détermine le niveau perçu de l'interdépendance entre le client et ses fournisseurs, est primordiale dans la fixation des objectifs imposés aux fournisseurs (principalement en termes de prix) et dans la mise en œuvre des sanctions (pénalités de retard, de non qualité, mise à l'écart pour de nouvelles consultations etc.).

Le contrôle opérationnel est orchestré par les acheteurs projet. Très structuré dans les deux organisations (jalons avec déclinaison des objectifs QCDP, étude des prototypes, confirmation de la pièce, mise en place des outillages et audits, remise des échantillons, plans de surveillance, accord de commercialisation), ce contrôle s'organise autour des revues technicoéconomiques mensuelles, des audits réguliers et d'une présence réciproque sur sites (fournisseurs présents sur les plateaux-projets, acheteurs projet présents auprès des fournisseurs). Bien que les dispositifs soient très formels, leur application conduit à la multiplication des interactions individuelles. Ces interactions facilitent la gestion fonctionnelle des conflits et font émerger une proximité relationnelle entre des acteurs qui s'apprécient et partagent les mêmes objectifs : démarrer la production du véhicule dans les délais et au niveau de qualité requis. Dès lors, le contrôle opérationnel stimule les échanges d'information, la solidarité, l'action jointe et la confiance qui, in fine, favorisent la cohésion interentreprises (Guibert et Dupuy, 1997). Le processus formel de contrôle implique ainsi un développement d'un mode de contrôle plus social, l'un et l'autre devenant complémentaires (Poppo et Zenger, 2002).

Les deux cas étudiés suggèrent l'enchevêtrement de deux modes de contrôle selon les phases de la relation. Les dispositifs formels sont de facture bureaucratique. Leur mise en œuvre implique une multiplication des interactions, un renforcement de la coopération et donc le développement d'un contrôle social (surtout au niveau des acheteurs projet). Cet enchevêtrement fait écho aux observations de Dyer (1996) dans l'industrie automobile américaine. En mettant en place un système très formel d'évaluation de la performance des fournisseurs (le système SCORE), Chrystler avait ainsi créé «par accident la méthode la plus 
efficace de l'entreprise pour créer la confiance [...] et améliorer la communication avec ses fournisseurs » (p. 49).

\subsection{Les cas Airindustrie et Pharmacop : une configuration de vassalité}

\subsubsection{Une dépendance asymétrique défavorable au client}

Airindustrie est la branche industrielle d'une compagnie aérienne. Elle est responsable de la maintenance et de l'entretien des avions. Ses fournisseurs lui livrent des pièces de mécanique, des systèmes électroniques ou des équipements pour les cabines. Ce sont des motoristes, des spécialistes de l'avionique ou des équipementiers aéronautiques. Tous les fournisseurs sont homologués par la Federal Aviation Administration (FAA). Tous sont des entreprises internationales, de taille supérieure ou comparable à Airindustrie. Peu nombreux (par ex. trois motoristes dans le monde), ils disposent de plus de 300 clients potentiels (des compagnies aériennes). Cette situation de petit nombre dans un contexte fortement réglementé implique qu'Airindustrie est dans une situation de dépendance asymétrique défavorable.

Pharmacop est un laboratoire pharmaceutique. Comme toute entreprise du secteur, Pharmacop travaille sur des cycles de développement très longs et est soumis à une réglementation stricte qui l'empêche de changer de formule et de processus de production une fois qu'un projet de médicament est homologué par les autorités. Dès lors, un choix initial de fournisseur ne peut quasiment pas être remis en cause par la suite. Les principaux fournisseurs de Pharmacop sont des grandes entreprises de chimie qui travaillent avec un grand nombre de laboratoires internationaux. Pharmacop est donc dans une situation de dépendance asymétrique défavorable.

Ces deux entreprises appartenant à deux secteurs différents sont finalement très dépendantes de leurs fournisseurs pour trois raisons : le petit nombre de fournisseurs potentiels, la réglementation qui limite leur changement et le caractère hautement technologique des produits et des process.

\subsubsection{Une structure achat décentralisée; un contrôle des acheteurs sur les méthodes} d'achat et les résultats logistiques

Airindustrie et Pharmacop ont une structure achat décentralisée qui permet aux acheteurs de travailler au plus près de leurs clients internes (la Direction Industrielle pour Airindustrie et la 
R\&D pour Pharmacop) ${ }^{7}$. Les décisions d'achat relèvent surtout de ces clients internes en charge de la spécification des technologies avec les fournisseurs. Bien qu'officiellement responsables des relations avec les fournisseurs, les acheteurs sont généralement intégrés très tard dans les processus de conception et d'industrialisation. Ils ne peuvent qu'entériner les choix de fournisseurs et s'assurer du bon approvisionnement des unités selon des plans décidés par les directions concernées. Face à leur situation de dépendance structurellement défavorable, l'impossibilité d'une négociation commerciale avec les fournisseurs s'inscrit dans les habitudes des acheteurs qui consultent les catalogues fournisseurs comme des bibles (d'après un acheteur travaillant auparavant dans le secteur automobile). Dès lors, la structure achat décentralisée qui place les acheteurs au plus près des prescripteurs internes est cohérente. La recherche d'une organisation parallèlement plus centralisée (comme dans les cas Consauto et Autoplus) paraîtrait coûteuse et inefficace au regard du pouvoir unilatéral des fournisseurs.

Le contrôle des acheteurs d'Airindustrie et de Pharmacop vise à asseoir leur rôle en interne : développer des méthodes d'achat et assurer la sécurité des approvisionnements en termes de qualite $^{8}$ et de délais. La direction souhaite "professionnaliser les achats ». Elle incite les acheteurs à prendre contact avec leurs fournisseurs, à contractualiser, à demander des justifications pour les hausses de prix, à développer un suivi logistique, etc. Le taux de contractualisation fait l'objet d'un suivi formel par indicateur. La direction achat souhaite également que les acheteurs soient informés des commandes passées. Pour cela, un indicateur sur «l'assiette » maîtrisée par chaque acheteur est mis en place.

Les dispositifs de contrôle ne visent pas l'obtention de résultats économiques, qui, compte tenu du rapport de force défavorable, seraient très limités. Airindustrie ne dispose pas d'indicateur fiable sur les gains sur achat. Pharmacop dispose d'un outil de suivi qui sert à vérifier le respect à + ou $-1 \%$ du budget fixé par les acheteurs eux-mêmes. L'évaluation des acheteurs est subjective; elle dépend de l'appréciation des comportements des acheteurs (disponibilité, sérieux, écoute, reconnaissance des autres, etc.) en interne et de leur capacité à résoudre les problèmes de logistique. 


\subsubsection{Une tentative de contrôle bureaucratique des fournisseurs qui se solde par un contrôle relationnel}

La sélection des fournisseurs ne repose pas sur des critères prédéfinis (qui n'existent pas) mais sur la base des expériences passées, de la réputation et de la confiance fondée sur les compétences techniques des fournisseurs connus depuis longtemps par les directions industrielles et R\&D. Sur les nouveaux projets, Airindustrie et Pharmacop activent leurs réseaux de connaissances, s'appuient sur leur relations interpersonnelles et promettent des conditions spéciales pour que les fournisseurs développent avec eux de nouveaux produits et process. Cette situation rappelle celle décrite par van der Meer-Kooistra et Vosselman (2000) sur les modalités du contrôle social. Une fois les fournisseurs sélectionnés, les acheteurs cherchent à établir des contrats, ce qui se situe dans la lignée des objectifs qui leur sont fixés. Cette recherche de contractualisation est conforme à la théorie des coûts de transaction dans les situations de petit nombre. Mais, conscients de leur force, les fournisseurs refusent les dispositions contractuelles qui pourraient leur être défavorables (engagement sur les prix, pénalités...). Ils acceptent uniquement les contrats imposés par les réglementations, les accords de confidentialité ou les contrats de fonctionnement «pour faire plaisir au client» avec des contraintes tellement lâches (par ex. pénalités en cas de retard de 180 jours) qu'elles ne font jamais l'objet d'application.

Dans la phase d'exécution, le processus de contrôle est peu structuré. Les prix sont négociés « au fil de l'eau ». Les seuls éléments de contrôle acceptés par les fournisseurs portent sur le respect de la qualité et des délais, considérés comme les «facteurs d'hygiène » de la coopération. Les fournisseurs ne donnent aucune information officielle sur leurs décompositions de coûts. Ils refusent tous les audits, visites ou autre forme d'observation intrusive. Dans les deux cas, des outils de pilotage objectifs et fiables n'ont pas été développés tant les acheteurs se considèrent dans l'incapacité d'exercer un quelconque contrôle formel sur des fournisseurs dominants. Chez Airindustrie, l'absence d'indicateurs fiables est parfois palliée par les fournisseurs eux-mêmes qui procurent leurs propres données (nombre de commandes, chiffre d'affaires, nombre de litiges, types de litiges, informations techniques, etc.) lorsque le self-audit est inscrit dans l'accord. De son côté, Pharmacop évalue chaque année ses fournisseurs de 1 à 5. L'évaluation est faite par chaque département (qualité, logistique, achat) mais, en l'absence de règles communes et explicites, la notation s'avère très subjective. Pour compenser le manque d'outils formels de contrôle des fournisseurs en phase 
d'exécution, Airindustrie et Pharmacop s'appuient à nouveau sur des modalités plus relationnelles. Les acheteurs cherchent à développer de bonnes relations personnelles, parfois même amicales avec leurs fournisseurs en multipliant les contacts, en se rendant disponibles et en présentant des perspectives de coopération future. Ce faisant, ils créent les conditions d'une « confiance affective » (McAllister, 1995). S'appuyant sur ce contrôle relationnel, les acheteurs tentent d'obtenir des fournisseurs une certaine flexibilité et une continuité dans les approvisionnements, aussi bien que des informations exclusives sur les nouveaux produits ou les concurrents et de favoriser la coopération. Leur réussite est valorisée par les directions R\&D et industrielle. Nos observations coïncident alors avec les travaux suggérant que la confiance de bonne volonté permet d'assurer la continuité des échanges (Sako, 1992), de favoriser l'engagement (Morgan et Hunt, 1994) et de susciter des normes de coopération (Larson, 1992). N'ayant pas de source objective de pouvoir, les acheteurs d'Airindustrie et de Pharmacop n'influencent pas leurs fournisseurs de manière coercitive. Ils leurs demandent parfois des explications et se proposent de les aider. La non coercition appelant la non coercition (Frazier et Rody, 1991), les fournisseurs sont enclins à privilégier les demandes des clients, ce qui ne préjuge pas de leur exécution.

\subsection{Le cas Telecom : une configuration de marché}

\subsubsection{Une faible interdépendance entre le client et le fournisseur}

Telecom est un équipementier en télécommunications qui conçoit et développe des équipements pour des opérateurs et le grand public. Les nombreux fournisseurs de Telecom sont de grandes entreprises d'envergure mondiale qui disposent d'un grand nombre de clients sur le marché global. Sur les produits standard, le remplacement d'un fournisseur peut être rapide et sans risque majeur. Sur les produits spécifiques, Telecom fait souvent travailler deux fournisseurs en parallèle pour ne pas perdre le temps du développement en cas de changement de fournisseur. .En définitive, Telecom et ses fournisseurs sont faiblement interdépendants.

\subsubsection{Une structure achat centralisée ; un contrôle des acheteurs sur les résultats et sur les comportements en interne}

La structure achat de Telecom est centralisée au niveau du groupe et des business units. Au niveau du groupe, les Commodity Manager Groupe (CMG) définissent, pour chaque famille 
de produits, une stratégie achat (choix des technologies, répartition des parts de marché globales entre les fournisseurs). Au niveau des business units, le directeur achat (qui rend compte au CMG) encadre des acheteurs qui aident à la définition de la stratégie, sélectionnent les fournisseurs et mènent les négociations sur le terrain. Cette structure centralisée est cohérente avec l'environnement achat de Telecom. Elle permet en effet de profiter des effets volume dans les négociations commerciales d'une part et de diminuer les coûts de structure d'autre part (Gadde et Hakansson, 1993).

Le contrôle des acheteurs de Telecom porte principalement sur les résultats, ce qui est cohérent avec l'environnement de faible interdépendance et la structure achat centralisée. Les acheteurs sont évalués sur les gains sur achats, les efforts logistique (cycle de livraison, gestion des stocks, flexibilité des fournisseurs) et sur la sécurisation des approvisionnements par l'introduction de nouvelles sources. Parmi ces critères, l'objectif économique est le plus valorisé. Pour obtenir des gains sur achat, les acheteurs s'appuient principalement sur la mise en concurrence périodique des fournisseurs par les prix. Cette stratégie peut engendrer des conflits en interne car les bureaux d'étude valorisent la technologie, les unités de production, la flexibilité logistique et les acheteurs recherchent les gains sur achats. Ces derniers doivent donc convaincre de la pertinence de leur stratégie. C'est pourquoi ils sont également évalués sur leur capacité à diffuser leur stratégie en interne et à montrer leur empathie vis-à-vis des clients internes (écoute des besoins, comportement positif, etc.). Conscient des injonctions contradictoires imposées aux acheteurs, Telecom a créé des postes de coordinateurs dont le rôle est de régler les conflits entre les différentes parties prenantes aux décisions d'achat.

\subsubsection{Un contrôle des fournisseurs par le marché tempéré par les résistances internes au sein de l'entreprise cliente}

De la sélection à l'exécution, Telecom cherche à tout moment à saisir les meilleures opportunités et exerce un contrôle par le marché tel que le décrivent van der Meer-Kooistra et Vosselman (2000). La sélection des fournisseurs est basée sur les offres techniques et commerciales (analyses des compétences technologiques, compétitivité, réactivité, flexibilité). Elle s'inscrit dans une stratégie achat décidée par chaque CMG qui donne les orientations en termes de choix de technologies et fournisseurs préférentiels. Les contrats proposés aux fournisseurs sont précis et explicites (prix, niveau de qualité, stocks tampon) pour les affaires dépassant un million d'euros. En deçà, l'accord conclu lors des négociations rapporté dans le 
compte-rendu a force exécutoire. Ils incluent à chaque fois une «road map » de décroissance des prix.

En phase d'exécution, les acheteurs reçoivent un rapport chiffré sur les résultats mensuels des fournisseurs en termes de qualité et de délais. Tous les trimestres, les fournisseurs sont notés (de 1 à 5) sur leur support opérationnel, commercial et technique. Les acheteurs ne disposent pas de la décomposition des coûts des fournisseurs. Mais, en consultant la concurrence, ils apprécient les niveaux de marge et fixent, sans trop d'erreurs, des objectifs de prix cibles. Lorsque les fournisseurs ne remplissent pas les conditions souhaitées, les acheteurs menacent de les remettre en concurrence. Dans les faits, la mise en application de ces menaces n'est pas aisée pour deux raisons: la faible dépendance des fournisseurs limite l'exercice d'une coercition; la résistance des clients internes (bureaux d'étude et unités de production) limite le changement de fournisseurs. En effet, tout changement de fournisseur nécessite de nouvelles adaptations techniques qui obligent les ingénieurs et opérateurs à modifier leurs process et engendre de nouvelles incertitudes. Dès lors, le contrôle par le marché voulu par les acheteurs et possible compte tenu de l'environnement achat de Telecom est entravé par des considérations internes à Telecom. Les acheteurs jugent leur tâche de négociation interne au moins aussi difficile que la négociation externe.

\subsection{Le cas Agro : une configuration de la seigneurie}

\subsubsection{Une dépendance asymétrique favorable au client}

Agro est un leader mondial de l'industrie agro-alimentaire qui achète des produits de base ou faiblement transformés (farines, produits sucrants, préparation de fruits, emballages, etc.). Ses fournisseurs sont très nombreux, non organisés collectivement et répartis sur toute la planète. Leur taille est bien inférieure à celle d'Agro qui contribue à une part importante de leur chiffre d'affaires. La situation de dépendance est très favorable à Agro ; elle lui confère un véritable pouvoir de négociation sur les fournisseurs.

\subsubsection{Une structure achat centralisée ; un contrôle des acheteurs sur les résultats et sur les comportements en interne}

La structure achat d'Agro est centralisée au niveau de chaque pôle d'activité et légère (pas d'acheteurs projet) ${ }^{9}$. Les produits communs à tous les pôles du groupe, sont sous la 
responsabilité de l'acheteur du pôle le plus consommateur qui achète pour l'ensemble du groupe. Les produits étant peu spécifiques et les fournisseurs nombreux, la structure centralisée permet, comme dans le cas Telecom, de profiter des effets volume tout en allégeant les coûts de structure. On retrouve d'ailleurs des mécanismes de contrôle des acheteurs similaires dans les deux entreprises.

Le contrôle des acheteurs porte principalement sur les résultats. Ils ont des objectifs ambitieux portant sur leur performance économique, la sécurité des approvisionnements, le respect des délais, de la qualité ${ }^{10}$ et le développement d'innovations. L'essentiel de leur évaluation porte sur leurs résultats en termes de gains sur achat. Un reporting mensuel permet de calculer les primes des acheteurs. Il repose sur trois types de données : des données sur l'évolution des prix du marché, les gains commerciaux et les gains de productivité. La réussite des acheteurs ne dépend pas seulement des gains issus des négociations commerciales (i.e., de leur pouvoir externe vis-à-vis des fournisseurs). Elle dépend aussi des gains issus des projets de productivité (e.g. un nouvel emballage moins coûteux). Cette double modalité d'évaluation des gains sur achat oblige les acheteurs à louvoyer entre les divergences internes opposant les usines (qui attendent la régularité et la qualité des livraisons des fournisseurs), les chefs de produits (qui freinent toute modification d'un produit rentable de peur d'en dégrader l'image et la performance) et leurs propres objectifs (de baisse de prix). Tout changement de fournisseur doit ainsi être justifié et accompagné de mesures favorisant, en interne, l'acception du changement : échanges directs avec les personnes, organisation de groupes de projet et de réflexion, visites d'usines, écoutes des attentes de chacune des parties prenantes et communications sur la stratégie achat etc. Ces comportements relationnels font également partie de l'évaluation des acheteurs.

\subsubsection{Un contrôle des fournisseurs par la coercition qui associe le marché et les dispositifs} bureaucratiques, mais tempéré par les stratégies de réseau des fournisseurs

La sélection des fournisseurs d'Agro repose officiellement sur cinq critères : la compétitivité, la qualité, l'innovation, la capacité à être global et la capacité à construire des relations dites «intégratives »(i.e. qui prennent en compte les services associés à la vente du produit). Toutefois, le prix est de loin le critère le plus important. Pour les produits standard, les enchères inversées sur internet sont un moyen particulièrement efficient pour obtenir le prix plancher. Les acheteurs d'Agro demandent à leurs fournisseurs de leur donner leur 
décomposition des coûts pour les mettre en concurrence sur chaque poste de leur structure de coûts. Toutefois, le choix final dépend beaucoup des usines, du département qualité et de la R\&D qui mettent en avant les fournisseurs qu'ils connaissent bien et qu'ils savent pouvoir répondre à leurs demandes spécifiques non inscrites dans le cahier des charges initial. Forts de leur pouvoir de négociation, les acheteurs demandent finalement au fournisseur préféré de l'usine ou de la R\&D de s'aligner sur les conditions de prix de la meilleure offre. Aucun contrat formel indiquant des objectifs précis n'est présenté aux fournisseurs. Agro attend de ces derniers une flexibilité totale et un comportement pro-actif dans la recherche de nouvelles sources de productivité.

En phase d'exécution, Agro contrôle ses fournisseurs de façon bureaucratique (quasihiérarchique). Les acheteurs redéfinissent régulièrement les objectifs en termes de prix, qualité, délais, volumes. Ils attendent également que les fournisseurs développent une stratégie (en termes d'innovation, d'organisation de la production...) en phase avec leurs demandes et imposent le respect de normes relationnelles à leur profit (rendre des services, être proactif dans la recherche de nouvelles sources de productivité, se montrer flexible, être totalement transparent sur les décompositions des coûts...). Les acheteurs disposent d'un tableau de bord mensuel par domaine d'achat, indiquant l'évolution du prix du marché, les gains sur les projets de productivité (optimisation des flux, modification du produit ou du process) et les gains commerciaux. Un reporting qualité mensuel ainsi qu'une synthèse des fiches d'incidents émises par les usines présente la performance qualité des fournisseurs. Chaque année, les fournisseurs sont notés de 1 à 5. Les fournisseurs défaillants ou mal notés sont sanctionnés (par une mise à l'écart temporaire ou une exclusion du panel). En parallèle de ces évaluations formelles, les acheteurs d'Agro s'immiscent chez les fournisseurs pour s'assurer d'être traités en clients privilégiés, vérifier in situ la véracité de leurs informations (en particulier les informations concernant les décompositions des coûts des fournisseurs, les parts de marché des différents clients, etc.) et maintenir une veille concurrentielle.

L'association du contrôle par le marché et du contrôle bureaucratique se traduit par une coercition qui trouve toutefois ses limites sur deux plans. D'une part, les acheteurs ne peuvent pas trop vite sacrifier les fournisseurs sur l'autel des prix afin de veiller à l'entretien d'un réseau étoffé de partenaires potentiels pour renforcer le poids de ses menaces. D'autre part, la coercition est contrainte par les résistances des autres acteurs internes. Ceux-ci préfèrent la stabilité à la rupture des relations pour plusieurs raisons : changer de fournisseur nécessite pour les usines de réaliser des nouveaux essais, ce qui occupe du «temps machine» 
improductif $^{11}$; le recours à un nouveau fournisseur accroît l'incertitude sur la qualité de l'approvisionnement; les opérationnels préfèrent traiter avec des fournisseurs qu'ils connaissent et dont ils savent qu'ils n'hésiteront pas à «donner un coup de main » en cas d'imprévu. Ceci renvoie au concept d'encastrement de Granovetter (1992, p. 498) pour qui, malgré la disponibilité des fournisseurs alternatifs, les individus « désirent éprouver un plaisir dans les interactions sociales qui accompagnent leur travail de tous les jours, ce qui serait complètement occulté [...] s'il fallait changer de partenaire sans arrêt». Ces réticences sont bien comprises par les fournisseurs d'Agro qui, ayant peu de leviers d'actions sur leur client, développent des stratégies actives d'immixtion au sein des unités de production (en étant présents auprès des opérateurs, des chefs d'atelier, des directeurs) et développent ainsi un réseau de relations permettant de susciter la bienveillance à leur égard. Ces stratégies semblent fonctionner. Les fournisseurs «préférés» des unités de production sont généralement sauvegardés par les acheteurs.

\section{Discussion et Conclusion}

Deux contributions principales se dégagent de cette recherche : (1) quatre configurations de contrôle client-fournisseur sont identifiées ; (2) la coexistence de plusieurs modes de contrôle pour une même configuration client-fournisseur est reconnue.

Notre première contribution est de proposer quatre configurations de contrôle clientfournisseur en fonction de l'environnement achat des clients. Le tableau 1 présente une synthèse des éléments constitutifs de chaque configuration. 


\begin{tabular}{|c|c|c|c|c|}
\hline CONFIGURATION & $\begin{array}{r}\text { PARTENARIAT } \\
\end{array}$ & VASSALITE & MARCHE & $\begin{array}{c}\text { SEIGNEURIE } \\
\end{array}$ \\
\hline $\begin{array}{l}\text { Situation de } \\
\text { dépendance dans } \\
\text { l'environnement } \\
\text { achat }\end{array}$ & $\begin{array}{l}\text { Une interdépendance forte entre } \\
\text { client et fournisseur }\end{array}$ & $\begin{array}{l}\text { Une dépendance asymétrique } \\
\text { défavorable au client }\end{array}$ & $\begin{array}{l}\text { Une interdépendance faible entre } \\
\text { client et fournisseur }\end{array}$ & $\begin{array}{l}\text { Une dépendance asymétrique } \\
\text { favorable au client }\end{array}$ \\
\hline $\begin{array}{l}\text { Organisation achat } \\
\text { du client }\end{array}$ & $\begin{array}{l}\text { Une structure achat matricielle } \\
\text { étoffée ; des fonctions support aux } \\
\text { achats } \\
\text { Des décisions d'achat relevant des } \\
\text { acheteurs en collaboration avec les } \\
\text { autres acteurs internes }\end{array}$ & $\begin{array}{l}\text { Une structure achat décentralisée } \\
\text { alignée sur les demandes internes } \\
\text { Des décisions d'achat relevant des } \\
\text { prescripteurs internes (ingénieurs } \\
\text { techniciens); des acheteurs } \\
\text { intégrés tard dans les processus de } \\
\text { décision }\end{array}$ & $\begin{array}{l}\text { Une structure achat centralisée } \\
\text { Des décisions d'achat nécessitant } \\
\text { un consensus entre les parties } \\
\text { prenantes à la relation client- } \\
\text { fournisseur (acheteurs, } \\
\text { prescripteurs, utilisateurs) }\end{array}$ & $\begin{array}{l}\text { Une structure achat centralisée } \\
\text { Des décisions d'achat nécessitant } \\
\text { un consensus entre les parties } \\
\text { prenantes à la relation client- } \\
\text { fournisseur (acheteurs, } \\
\text { prescripteurs, utilisateurs) }\end{array}$ \\
\hline $\begin{array}{l}\text { Contrôle des } \\
\text { acheteurs }\end{array}$ & $\begin{array}{l}\text { Un contrôle des acheteurs formel et } \\
\text { structuré portant sur la stratégie } \\
\text { achat, les résultats (principalement } \\
\text { économiques), les procédés de } \\
\text { travail et les comportements à } \\
\text { l'égard des fournisseurs }\end{array}$ & $\begin{array}{l}\text { Un contrôle des acheteurs portant } \\
\text { sur le développement de méthodes } \\
\text { d'achat et sur les résultats } \\
\text { logistiques (non économiques); } \\
\text { une volonté de professionnaliser les } \\
\text { achats et d'asseoir leur rôle en } \\
\text { interne }\end{array}$ & $\begin{array}{l}\text { Un contrôle des acheteurs portant } \\
\text { sur les résultats (principalement } \\
\text { économiques) et sur les } \\
\text { comportements internes (capacité } \\
\text { de conviction sur la stratégie } \\
\text { d'achat, d'écoute) }\end{array}$ & $\begin{array}{l}\text { Un contrôle des acheteurs complet } \\
\text { et ambitieux portant sur les } \\
\text { résultats (principalement } \\
\text { économiques) et sur les } \\
\text { comportements internes (capacité } \\
\text { de conviction sur la stratégie } \\
\text { d'achat, d'écoute) }\end{array}$ \\
\hline $\begin{array}{l}\text { Contrôle des } \\
\text { fournisseurs }\end{array}$ & $\begin{array}{l}\text { Un contrôle bureaucratique } \\
\text { structuré : systèmes formels } \\
\text { d'information, intervention } \\
\text { directe ; audit des process } \\
\text { Un contrôle social issu de la } \\
\text { multiplication des interactions liée } \\
\text { à la mise en œuvre du contrôle } \\
\text { bureaucratique }\end{array}$ & $\begin{array}{l}\text { Une tentative de contrôle } \\
\text { bureaucratique qui échoue en } \\
\text { l'absence d'indicateurs précis et } \\
\text { fiables et en l'absence de pouvoir } \\
\text { de négociation } \\
\text { Un contrôle social issu des bonnes } \\
\text { relations interpersonnelles à } \\
\text { l'initiative des acheteurs }\end{array}$ & $\begin{array}{l}\text { Un contrôle par le marché : mise en } \\
\text { concurrence périodique, évaluation } \\
\text { formelle et régulière sur le soutien } \\
\text { opérationnel et commercial } \\
\text { Une application du contrôle par le } \\
\text { marché tempéré par les résistances } \\
\text { internes d'acteurs préférant la } \\
\text { continuité à la rupture des relations }\end{array}$ & $\begin{array}{l}\text { Un contrôle par la coercition qui } \\
\text { associe le marché et les dispositifs } \\
\text { bureaucratiques : mise en } \\
\text { concurrence ; systèmes formels et } \\
\text { informels d'information; exigence } \\
\text { de soumission aux demandes des } \\
\text { prescripteurs et utilisateurs } \\
\text { Une coercition tempérée par la } \\
\text { nécessaire sauvegarde d'un réseau } \\
\text { de fournisseurs et par les } \\
\text { résistances internes renforcées par } \\
\text { les stratégies d'immixtion } \\
\text { relationnelle des fournisseurs }\end{array}$ \\
\hline
\end{tabular}

Tableau 1 : Les configurations de contrôle client-fournisseur 
Pour chaque configuration, nous observons une grande cohérence entre l'environnement achat, l'organisation du client et les modalités de contrôle client-fournisseur. Trop de chercheurs considèrent les entreprises comme des boîtes noires dont les conséquences des problématiques internes sont négligées.

Cette recherche révèle au contraire l'existence d'un fit entre l'organisation achat d'une entreprise et l'exercice de son contrôle sur ses fournisseurs. Prenons le cas de la seigneurie. L'environnement achat du client lui est très favorable. Dès lors, l'organisation achat est centralisée pour profiter des effets de volume et asseoir la domination du client dans les négociations. En interne, le contrôle des acheteurs est naturellement axé sur des résultats économiques ambitieux. Mais, compte tenu de la participation des autres acteurs internes aux décisions d'achat (prescripteurs, utilisateurs), le contrôle des acheteurs est également axé sur leurs comportements, en particulier sur leur capacité à communiquer sur leur stratégie achat et à intégrer, dans cette stratégie, les besoins de ces acteurs. Ces deux aspects du contrôle des acheteurs (gains économiques et comportements en interne) ont une influence sur le contrôle que les acheteurs mettent ensuite en œuvre auprès de leurs fournisseurs : le contrôle (à la fois par le marché et bureaucratique permis par le fort pouvoir du «seigneur ») est coercitif et permet aux acheteurs d'obtenir les meilleurs prix du marché. Toutefois, ce contrôle coercitif se trouve tempéré par les résistances internes et, finalement, les acheteurs conservent généralement les fournisseurs préférés par les acteurs internes.

Cette cohérence dans la configuration de la seigneurie entre l'environnement achat, l'organisation du client (structure achat et contrôle des acheteurs) et le contrôle sur les fournisseurs s'observe également dans les autres configurations. Dès lors, il nous semble important que les modèles de contrôle interorganisationnel intègrent systématiquement les problématiques du contrôle intraorganisationnel. Nous avons commencé par intégrer celles concernant le contrôle intraorganisationnel des acheteurs. Il conviendrait d'approfondir cette réflexion sur les différentes parties prenantes aussi bien dans les entreprises clientes que chez les fournisseurs.

Les configurations proposées rappellent aussi l'influence de la dépendance dans l'exercice des relations commerciales entre des entreprises clientes et leurs fournisseurs, ce qui avait été occulté par les chercheurs focalisant leurs études sur la coopération et le développement mutuel. Même dans les relations les plus équilibrées et les partenariats, il existe des rapports de force que les partenaires d'échange n'hésitent pas à exploiter. Cette observation va à 
l'encontre des discours «collaboratifs» prononcés lors des annonces formelles de coopérations interentreprises mais elle reflète la réalité de l'exercice du contrôle interorganisationnel. Nous invitons donc les chercheurs à mieux considérer l'amplitude et l'asymétrie de la dépendance des acteurs d'une relation avant d'en analyser son fonctionnement et ses enjeux. Certains chercheurs en marketing Business to Business l'ont déjà compris (Berthon et al., 2003).

La seconde contribution concerne l'observation d'un enchevêtrement de plusieurs modes de contrôle pour une même configuration client-fournisseur. Cette observation vient confirmer les propositions théoriques de Bradach et Eccles (1989). Elle vient aussi conforter les résultats de recherches empiriques sur le contrôle inter-organisationnel : l'étude de Baglin et Malleret (1995) sur les modes de gestion de la performance dans les chaînes hôtelières ou celle de Froehlicher (2000) dans une entreprise d'électricité. La configuration du partenariat en est un bon exemple. La structure achat étoffée dans cette configuration permet de différencier les niveaux de relations (le niveau des acheteurs par famille et le niveau des acheteurs par projet). A ces deux niveaux, les mécanismes de contrôle sont formels et bureaucratiques. Toutefois, les sujets traités diffèrent. Les négociations commerciales, parfois tendues, se gèrent principalement au niveau des acheteurs par famille; les relations techniques se gèrent au niveau des projets. Entre les équipes projet, la multiplication des interactions dans un contexte de coopération (sans problème de prix) permet le développement d'un mode de contrôle social. La complémentarité entre le contrôle formel (bureaucratique) et le contrôle social fait écho aux travaux de Guibert et Dupuy (1998) et à ceux de Poppo et Zenger (2002). Cet enchevêtrement de différents modes de contrôle aux différents niveaux de la relation peut être un gage de performance des relations client-fournisseur. Comme Uzzi (1997) le soulignait à propos des réseaux d'entreprises, un réseau est performant s'il est composé de liens encastrés et de liens plus lâches. Nous faisons la même observation dans les relations clientfournisseur: des relations encastrées permettent aux individus de coopérer sur un projet commun. Parallèlement, des relations plus tendues (par ex. au niveau des acheteurs famille) procurent la compétition nécessaire à la progression mutuelle. Coopération et compétition sont donc imbriquées dans une même relation client-fournisseur selon les acteurs impliqués.

En ne considérant plus les entreprises comme des boites noires, cette recherche fait ainsi apparaître les véritables enjeux des relations client-fournisseur. Ces enjeux sont beaucoup plus étendus que de simples relations marchandes entre un acheteur et un commercial. 
Les limites de cette recherche sont principalement liées aux choix méthodologiques. Bien que les configurations proposées soient riches d'enseignements, elles devront être confrontées à d'autres études et testées dans d'autres contextes. Aussi, nous avons focalisé nos observations sur les équipes achat d'entreprises clientes, considérant que ces équipes étaient les responsables officiels des relations client-fournisseur. Une analyse plus approfondie des autres parties prenantes (en particulier les prescripteurs et les utilisateurs) sur les décisions d'achat serait néanmoins riche d'enseignements pour des recherches futures. Enfin, la dynamique des relations client-fournisseur mériterait un traitement plus fin. Nous avons certes distingué les deux principales phases d'une relation client-fournisseur (la sélection et l'exécution) mais il faut aller plus loin. L'environnement achat (ici la structure de la dépendance) d'une entreprise peut évoluer, de même que son organisation interne. Pour comprendre ces évolutions, des études longitudinales sembleraient pertinentes et permettraient d'améliorer la portée du modèle. 


\section{Références bibliographiques}

Anderson J.C., Hakansson H., Johanson J. (1994), « Dyadic business relationships within a business network context », Journal of Marketing, vol. 58, Octobre, pp. 1-15.

Anthony R.N. (1993), La fonction contrôle de gestion, Paris, Publi-Union.

Aoki M. (1991), Economie japonaise, Paris, Economica.

Baglin G. et Malleret V. (1995), «Les modes de gestion de la performance dans les chaînes hôtelières françaises », Revue Française de Comptabilité, n²68, juin, pp. 47-56.

Berthon P., Pitt L.F, Ewing M.T, Bakkeland G. (2003), « Norms and power in marketing relationships: Alternative theories and empirical evidence », Journal of Business Research, vol. 56, n9, pp. 699-710.

Blau M. P. (1964), Exchange and Power in Social Life, New York, John Wiley \& Sons.

Bouquin H. (2006), Le contrôle de gestion, Paris, PUF, $7^{\mathrm{ème}}$ éd.

Bradach J.L. et Eccles R.G. (1989), «Price, authority and trust: from ideal types to plural forms », Annual Review of Sociology, Vol. 15, pp. 97-118.

Celly K.S., Frazier G.L. (1996), «Outcome-Based and Behavior-Based Coordination Efforts in Channel Relationships », Journal of Marketing Research, vol. 33, May, pp. 200-210.

Cox A. (2004), Win-win? The paradox of value and interests in business relationships, Boston, Earlsgate Press.

Dekker H.C. (2004), « Control of inter-organizational relationships: evidence on appropriation concerns and coordination requirements », Accounting, Organizations and Society, vol. 29, pp. 27-49.

Desreumaux A. (1992), Structures d'entreprises, Paris, Vuibert gestion.

Donada C., Nogatchewsky G. (2006), « Vassal or lord buyers : How to exert management control in asymmetric interfirm transactional relationships ?», Management Accounting Research, vol. 17, n³, pp. 259-287.

Dumoulin R. (1997), «Marché, bureaucratie et clans dans les réseaux stratégiques », Comptabilité Contrôle Audit, tome 3, vol. 2, pp. 45-58.

Dumoulin R., Gbaka A. (1997), «Contrôle d'entreprises et réseaux stratégiques, une étude exploratoire », Comptabilité Contrôle Audit, tome 3, vol. 1, pp. 23-38.

Dwyer F., Schurr P., Oh S. (1987), «Developing Buyer-Seller Relationships », Journal of Marketing, vol. 51, pp. 11-27.

Dyer J.H. (1996), « Specialized supplier networks as a source of competitive advantage: Evidence from the auto industry », Strategic Management Journal, vol. 17, n4, pp. 271-291.

Emerson T.M. (1962), « Power-Dependence Relations », American Sociological Review, vol. 27, pp. 31-41.

Fabre P. (2006), « Mairies et associations partenaires : l'impact des relations interpersonnelles sur le processus de contrôle », Humanisme et Entreprises, n²79, octobre. 
Flamholtz E.G. (1996), «Effective organizational control: A framework, applications and implications », European Management Journal, vol. 14, nº, pp. 596-611.

Frazier G.L., Gill J.D., Kale S.H. (1989), « Dealer Dependence Levels and Reciprocal Actions in a Channel of Distribution in a Developing Country », Journal of Marketing, vol. 53, January, pp. 50-69.

Frazier G.L., Rody R.C. (1991), «The use of influence strategies in interfirm relationships in industrial product channels », Journal of Marketing, vol. 55, n 1, pp. 52-69.

Froehlicher T. (2000), «La dynamique de l'organisation relationnelle : conventions et réseaux sociaux au regard de l'enchevêtrement des modes de coordination », Finance Contrôle Stratégie, vol 3, n² 2, pp. 113-143.

Gadde L.-E., Hakansson H. (1993), Professional Purchasing, London, Routledge.

Granovetter M. (1992), «Problems of Explanation in Economic Sociology », in Nohria N., Eccles R. (ed.), Networks and organizations: Structure, form and action, Boston, Harvard Business School Press, pp. 25-56.

Guibert N. et Dupuy Y. (1997), «La complémentarité entre contrôle « formel » et « contrôle informel »: le cas de la relation client-fournisseur », Comptabilité Contrôle Audit, tome 3, vol. 1, pp. 39-52.

Gundlach G.T., Cadotte E.R. (1994), «Exchange interdependence and interfirm interaction: research in a simulated channel setting », Journal of Marketing Research, vol. 31, n4, pp.516-532.

Hakansson H., Lind J. (2004), «Accounting and network coordination », Accounting, Organizations and Society, vol. 29, pp. 51-72.

Heide J.B. (1994), «Interorganizational Governance in Marketing Channels », Journal of Marketing, vol. 58, pp. 71-85.

Jap S. Ganesan S. (2000), «Control mechanisms and the relationship life cycle : Implications for safeguarding specific investments and developing commitment », Journal of Management Research, vol. 37, n², pp. 227245.

Jensen M.C., Meckling W.H. (1976), « Theory of the Firm : Managerial Behavior, Agency Costs and Ownership Structure », Journal of Financial Economics, vol. 3, pp. 306-360.

Kim K. (2000), « On interfirm power, channel climate, and solidarity in industrial distributor-supplier dyads », Journal of the Academy of Marketing Science, vol. 28, n³, pp. 388-405.

Langfield-Smith K., Smith D. (2003), « Management control systems and trust in outsourcing relationships », Management Accounting Research, vol. 14, pp. 281-307.

Larson A. (1992), «Network Dyads in Entrepreneurial Settings: A Study of Governance of Exchange Relationships », Administrative Science Quarterly, vol. 37, pp. 76-104.

Lusch R.F., Brown J.R. (1996), «Interdependency, Contracting and Relational Behavior in Marketing Channels », Journal of Marketing, vol. 60, October, pp. 19-38.

Macaulay S. (1963), «Non-Contractual Relations in Business : a Preliminary Study », American Sociological Review, vol. 28, February, pp. 55-67. 
Macneil I.R. (1980), The New Social Contract, An Inquiry into Modern Contractual Relations, CT: Yale University Press.

McAllister D.J. (1995), «Affect- and cognition-based trust as foundations for interpersonal cooperation in organizations », Academy of Management Journal, vol. 38, pp. 24-59.

Miles M., Huberman A. (1991), Analyse de données qualitatives, recueil de nouvelles méthodes, Bruxelles, De Boeck Université (1ère édition américaine 1984).

Mintzberg H. (1982), Structure et dynamique des organisations, Paris, Ed. d'Organisation.

Morgan R., Hunt S. (1994), «The Commitment-Trust Theory of Relationship Marketing », Journal of Marketing, vol. 58, July, pp. 20-38.

Ouchi W.G. (1980), «Markets, bureaucracies and clans », Administrative Science Quarterly, vol. 25, n¹, pp. $129-141$

Petitjean J-L. (2001), «Coordination inter-firmes : de la différenciation des configurations organisationnelles à l'intégration des mécanismes de contrôle », Actes du XXIIème congrès de la l'AFC, Metz, mai.

Pezet A. (2001), «Reconfigurer la décision d'investissement stratégique : du processus au réseau encastré », Economies et Sociétés, série « Sciences de Gestion », n³0, automne, pp. 59-82.

Pfeffer J. (1981), Power in organizations, Boston, Pitman.

Pfeffer J., Salancik G. (1978), The External Control of Organizations, New York, Harper \& Row Publishers.

Poppo L., Zenger T. (2002), «Do formal contracts and relational governance function as substitutes or complements ?», Strategic Management Journal, vol. 23, pp. 707-725.

Ring P.S., Van de Ven A.H. (1992), «Structuring Cooperative Relationships between Organizations », Strategic Management Journal, vol. 13, pp. 483-498.

Ring P.S., Van de Ven A.H. (1994), «Development Processs of Cooperative Interorganizational Relationships », Academy of Management Review, vol. 19, n¹, pp. 90-118.

Sako M. (1992), Price, Quality and Trust. Interfirm Relations in Britain and Japan, Cambridge, Cambridge University Press.

Thibaut J.W., Kelley H.H. (1959), The Social Psychology of Groups, New York, John Wiley.

Ulrich D., Barney J.B. (1984), «Perspectives in Organizations: Resource Dependence, Efficiency and Population », Academy of Management Review, vol. 9, n³, pp. 471-481.

Uzzi B. «Social structure and competition in interfirm networks: The paradox of embeddedness », Administrative Science Quarterly, vol. 42, n¹, pp. 37-69.

Van der Meer-Kooistra J., Vosselman G.J. (2000), «Management control of interfirm transactional relationships: The case of industrial renovation and maintenance », Accounting, Organizations and Society, vol. 25, pp. 51-77. 
Williamson O.E. (1975), Markets and hierarchies: Analysis and antitrust implications, New York, the Free Press.

Williamson O.E. (1985), The Economic Institutions of Capitalism, New York, The Free Press. Traduction française (1994), Les institutions de l'économie, InterEditions.

Yin R.K. (1989), Case study research : design and methods, Newbury Park, Sage Publications, $2^{\text {nde }}$ édition.

\begin{abstract}
${ }^{1}$ Nous regroupons sous le terme «contrôle social » les modes de contrôle qui reposent sur la confiance et le respect des normes relationnelles.

${ }^{2}$ Certains auteurs s'appuient sur les travaux d'Ouchi (1980) et proposent une application de son modèle aux relations inter-organisationnelles. Ce modèle s'inscrit dans la lignée des approches transactionnelle et relationnnelle. Le marché et la bureaucratie sont une adaptation organisationnelle des travaux de Williamson (1975). Le clan est un concept très proche de celui de l'échange relationnel (Heide, 1994) : ils reposent tous deux sur des normes selon lesquelles les objectifs individuels ne peuvent être atteints que par l'action jointe.
\end{abstract}

${ }^{3}$ Les termes «vassalité » et «seigneurie» ont été choisis pour illustrer deux situations de dépendance asymétrique. Le mot vassal vient du latin vassus serviteur. Dans ce système féodal, seigneur et vassal sont certes liés par des obligations réciproques mais le seigneur dispose du droit de ban. Au XVIII ${ }^{e}$ siècle, un vassal désigne un simple paysan libre mais entièrement dépendant de celui qui le fait travailler.

${ }^{4} \mathrm{PPM}=$ (nombre de pièces avec défauts) $/$ (nombre total de pièces) $* 1000000$

${ }^{5}$ Chaque critère est sous la responsabilité d'une des parties prenantes. Le département qualité note la qualité, la direction de l'analyse des coûts note la compétitivité, les experts notent la présence internationale, la gouvernance, la logistique et l'industrialisation et enfin, les acheteurs notent le climat relationnel.

${ }^{6}$ Le terme de confiance renvoie ici à l'anticipation sur le fait que les attentes ne seront pas déçues. Les chercheurs de l'approche de l'échange relationnel ont une conception plus restreinte de la confiance, confiance qui repose sur la foi dans la bonne volonté de l'autre (Ring et van de Ven, 1992).

${ }^{7}$ Les acheteurs de Pharmacop ont longtemps été hiérarchiquement rattachés à la R\&D, ceux d'Airindustrie à la direction industrielle. Récemment les deux entreprises ont décidé de redonner aux acheteurs l'entière responsabilité de toute la relation avec les fournisseurs (définition de la politique d'achat, participation à la sélection des fournisseurs, élaboration des contrats, négociation ex ante des prix, définition des plans de performance, pilotage et évaluation des fournisseurs) en créant une direction des achats indépendante. Toutefois, les réflexes de soumission hiérarchique demeurent à l'égard des autres directions.

${ }^{8}$ Bien qu'essentielle à la réussite des projets aéronautiques et pharmaceutiques, la qualité n'est évaluée que par la Direction Qualité des entreprises.

${ }^{9}$ Les audits d'homologation des fournisseurs sont assurés par les agents de l'assurance qualité assistés par les Responsables Achat.

${ }^{10}$ La qualité est certes un élément important dans le cas Agro, mais elle est surtout gérée par la direction de la sécurité alimentaire et finalement assez peu par les acheteurs (excepté pour certains cas sensibles comme l'achat de lait ou de fruits.

${ }^{11}$ Les usines d'Agro sont notées, entre autre, sur leur taux de rendement synthétique. Tout temps improductif implique une baisse de cet indicateur. 